\title{
Analysis of Extracted Forearm sEMG Signal Using LDA, QDA, K-NN Classification Algorithms
}

\author{
Firas AlOmari* and Gunhai Liu
}

Jiangsu University-School of Electrical and Information Engineering, Xuefu Rd 301\#, Zhenjiang 212013, PR China

\begin{abstract}
A surface electromyographic (sEMG) signal includes important information on muscular activity and was recently widely used as an input signal in a myoelectric control system. In this manuscript, eight hand motions were classified using different extracted features from sEMG signals. The results of the experiment show that the combination of sample entropy (SampEnt), root mean square (RMS), myopulse percentage rate (MYOP), and difference absolute standard deviation value (DASDV) achieved the highest classification rate of $98.56 \%$ using the linear discriminant analysis (LDA) classifier. Moreover, this study investigated the best value of $\mathrm{K}$ that should be used as an input parameter in the $\mathrm{K}$-nearest neighbor $(\mathrm{K}-\mathrm{NN})$ algorithm. The result demonstrates that $\mathrm{k}=5$ is the optimal choice in most cases.
\end{abstract}

Keywords: EMG, feature extraction, human-machine interface, myoelectric control system, pattern recognition, signal processing, wavelet analysis.

\section{INTRODUCTION}

A prosthetic hand becomes a valuable artificial replacement to amputees because it can help to restore some of the capabilities of their lost hand. The classification accuracy of a myoelectric control system depends on many factors: the position and number of electrodes, which are responsible for the collection of the sEMG signals; the choice of an effective classifier that can differentiate the recorded sEMG signals; and feature weighting. In a myoelectric control system, feature weighting is a method used to extract useful information from sEMG signals and to use this information, which represents the recorded signal, to classify different motion patterns. In the following section, we present some of the significant works related to classification of sEMG signals using different recognition algorithms.

Khushaba and his colleagues [1] suggested a muscle interface system to help reduce driver distraction. Different extracted features were extracted to classify 14 different finger postures by implementing four different classifiers, LIBSVM, LDA, REGTREE and NaiveBayes. In addition, they proposed the fuzzy neighborhood discriminant (FNDA), a method for discriminant feature extraction and to solve the channel selection problem.

Three wavelet families (Haar, db, and sym) at different decomposition levels were tested by Kumar et al. [2], who found that the use of sym 4 and sym5 at the decomposition rates 8 and 9 can obviously distinguish between sEMG signals related to fatigued and non-fatigued muscles.

Hong-Bo et al. [3] proposed a swarm intelligence-based sEMG feature selection algorithm to classify eight different

*Address correspondence to this author at the Jiangsu University-School of Electrical and Information Engineering, Xuefu Rd 301\#, Zhenjiang 212013, PR China; Tel: 008615806109605; E-mail: fomari6@gmail.com hand movements. Ant colony optimization (ACO) and the minimum redundancy maximum relevance criterion (mRMR) were used. They extracted two sets of features from the sEMG signals in the time domain and the timefrequency domain and used a back propagation neural network as a classification method. They found that the average number of features selected in the optimum set was 9.9 for the six selected time domain features and autoregressive coefficients combination, and 9.3 for wavelet coefficients. They achieved a $96.08 \%$ average classification rate using the extracted wavelet coefficient.

Kilby and Gholam [4] collected sEMG signals from a muscle under sustained contractions for a period of four seconds using different loads and then analyzed the signal using fast Fourier transform (FFT), discrete wavelet transform (DWT), and wavelet packet transform (WPT). Based on their study, it is recommend the use of Daubechies, Symmlet, and Coiflet families for sEMG analysis.

Another research group [5] compared the performance of different wavelet families. Two types of radial neural networks, GRNN and probability neural network (PNN) were implemented. To classify five types of hand motions, they tested four wavelet families (bior, coif, $\mathrm{db}$ and sym) at different decomposition levels. Based on their study, the Biorthogonal and Coiflets wavelet families performed better than other wavelet families. They also found that the GRNN classifier achieved the highest average classification accuracy based on the coif wavelet family.

In this manuscript, different features were extracted from a sEMG signal and analyzed using three classification algorithms LDA, quadratic discriminant analysis (QDA), and $\mathrm{k}-\mathrm{NN}$. The selected features were SampEnt, RMS, MYOP, DASDV, Wilson amplitude (WAMP), and wavelet coefficients (WAVELET). We also combined some features to obtain the optimal result with the highest classification accu- 
Table 1. Data acquisition system and related channels responsible for the collection of sEMG signals.

\begin{tabular}{|c|c|c|}
\hline \multirow{2}{*}{ DAS } & Channel Number & Muscle \\
\hline \hline \multirow{2}{*}{1 1st } & 1 & extensor digitorum \\
\cline { 2 - 3 } & 2 & extensor carpi radialis \\
\hline \multirow{2}{*}{ 2nd } & 3 & palmaris longus \\
\cline { 2 - 3 } & 4 & flexor carpi ulnaris \\
\hline
\end{tabular}

racy. The tested feature combinations were SampEnt-RMS, SampEnt-RMS-MYOP, and SampEnt-RMS-MYOP-DASDV.

The remainder of this manuscript is divided into five sections. The first gives the reader information on the experimental protocol. The second section provides a brief introduction to features extraction and presents wavelet analysis technique. The third section introduces the classification algorithms that were used in this research study. The last two sections present and discuss the results obtained from the experiment and present the conclusions drawn from the results and suggestions for future work, respectively.

\section{EXPERIMENT AND DATA ACQUISITION}

\subsection{Experimental Design}

Ten right hand-dominant healthy subjects (males aged from 20 to 37 years) without any neuromuscular disorders participated in this experiment. The subjects were well trained on all of the movements before performing the hand motions and were given a demonstration of the process of this research work. At the end of the demonstration, the subjects were not afraid of the equipment, cables, and attached electrodes connected to them.

Before starting the experiment, all of the subjects were asked to rest before participating in the experiment to ensure a lack of stress and physical pressure during the experiment that may affect the results. In addition, a good and proper environment was provided to ensure the collection of accurate and less-noisy sEMG signals. The data were recorded over a period of ten days to affirm different realistic conditions. To collect four sEMG signals from the forearm muscles, two pieces sEMG signal recording equipment (AD Instrument's Power Lab 4/25 T) were used. Each data acquisition system (DAS) has two channels, in total, four sEMG signals were acquired from four different forearm muscles, as clarified in Table $\mathbf{1}$.

The surface electromyographic signal (EMG) is influenced by several physiological and anatomical factors [6]. Electromagnetic fields caused by a power line represent a common noise source in the EMG signal recorded from the body surface. Such noise is characterized by $50 \mathrm{~Hz}$ sinusoidal interference, possibly accompanied by a number of harmonics [7]. However, a bandpass filter with a $10-$ to $500-\mathrm{Hz}$ bandwidth, a 50-notch filter and a mains filter were used.
The data were sampled at $1 \mathrm{KHz}$. All of the data were normalized and segmented into consecutive 256-ms epochs, as clarified in Fig. (1).

Disposable moisture silver-silver chloride $(\mathrm{Ag} / \mathrm{AgCl})$ electrodes were used to obtain the sEMG signals from the surface of the skin. The unipolar electrodes were placed along the midline of the muscle and parallel to the longitudinal axis of the muscle to prevent signal crosstalk between adjacent muscles, and to ensure that the detection will intersect common muscle fibers. In this paper, we attempted to recognize the eight hand movements clarified in Fig. (2).

\subsection{Experimental Protocol}

All of the subjects were asked to perform eight movements. Each movement/action was repeated five times, and each action was held for five seconds. In addition there was a rest period of approximately three seconds between each set of two hand motions.

After finishing one movement, the subject rested for almost three minutes to avoid muscle fatigue. During this time, the collected data were saved, and the subject prepared himself for the next movement. The four sEMG signals were collected and arranged together to form a matrix denoted raw Matrix (RM) as clarified in Fig. (3). In addition, it is clear that the amplitude and shape of each EMG signal are different because the muscles exerted different forces during the performance of the different hand motions.

\section{FEATURE EXTRACTION}

In a myoelectric control system, some extracted features represent the sEMG signal better than the other features [8]. There are many potential benefits of feature weighting, the first one is to reduce the dimensionality of the row dataset which help reducing the storage requirements, in addition to reduce training time of classifier which lead to practical prosthetic hand that mimicking the real hand [9]. Every extracted feature will be introduced to the classifier will give different classification rate some lead to better result in comparison to other features.

It is meaningful to look for the feature that gives the optimal representation of the sEMG. Some features extracted from sEMG signal based on frequency domain are not good in EMG signal classification. Some frequency domain features have the same classification accuracy as features in time domain [8]. Features in time domain are the most popular 

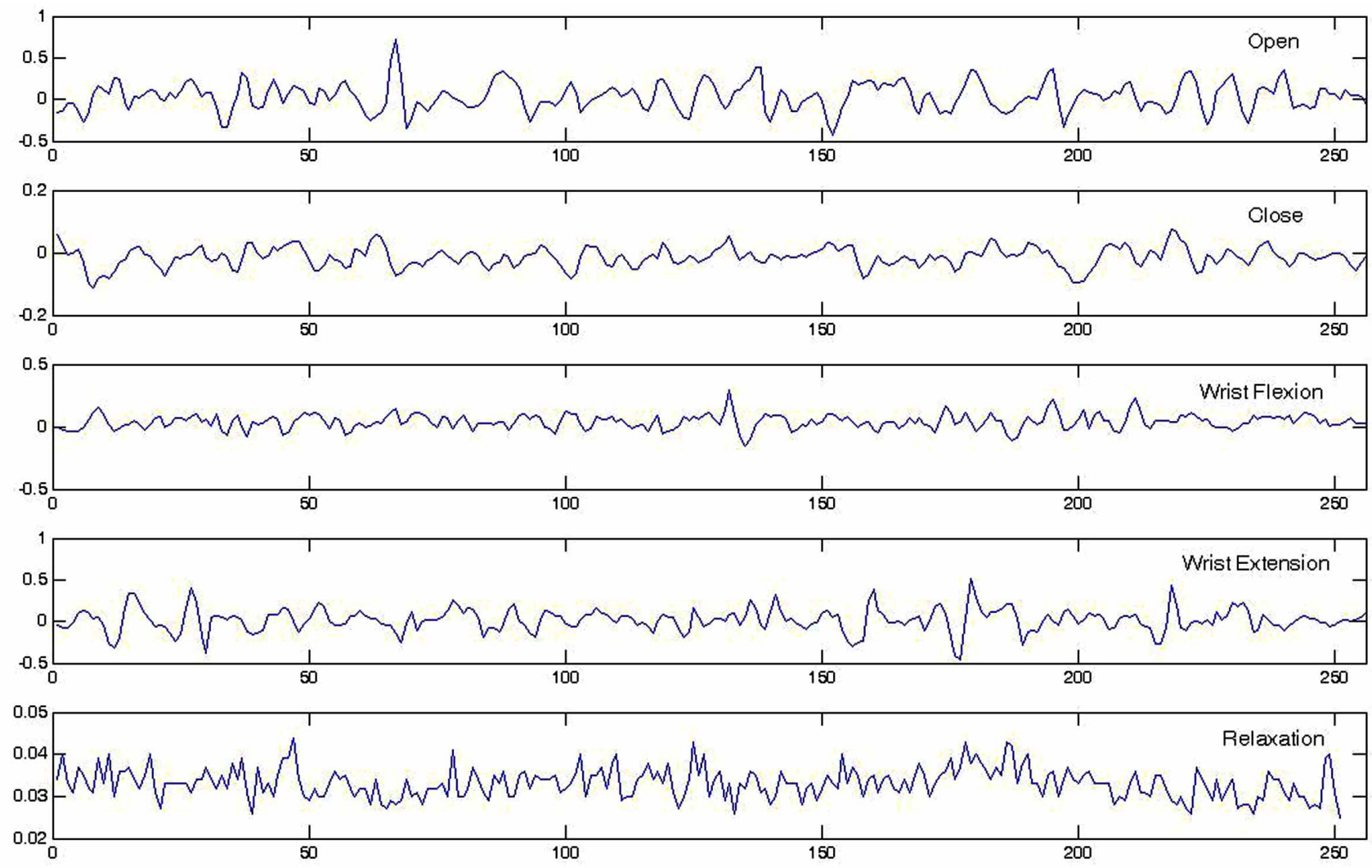

Fig. (1). Segmented sEMG signal in 256 points window for one subject performing four hand motions (Open hand, close hand, wrist flexion and wrist extension) and rest state.
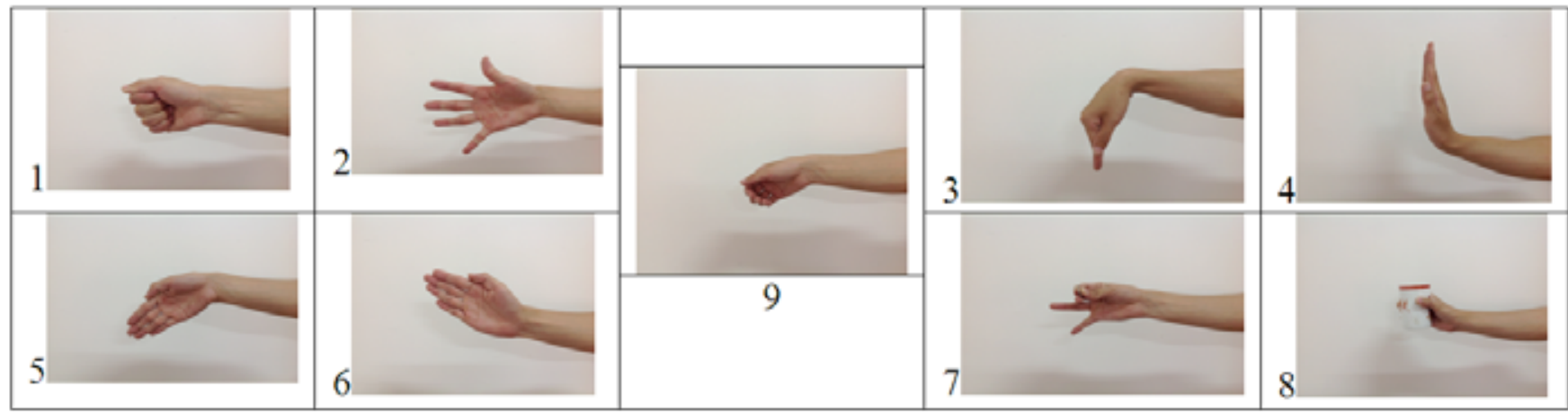

Fig. (2). Eight classified hand motions: 1- grip (GP), 2- open hand (OP), 3- wrist flexion (WF), 4- wrist extension (WE), 5- ulnar deviation (UD), 6-radial deviation (RD), 7- pinch (PIN), 8- catch cylindrical subject (CC), and 9- rest position (REST).

in the field of myoelectric pattern recognition [9]. They are easy to implement, because these features do not need any transformation [8]. Many research works explored and examined appropriate qualitative and quantitative EMG signal features

In this manuscript, different features were extracted from a sEMG signal and analyzed using three classification algorithms. We also combined some features to obtain the optimal result with the highest classification accuracy. The tested feature combinations were SampEnt-RMS, SampEnt-
RMS-MYOP, and SampEnt-RMS-MYOP-DASDV. Firstly, a short explanation was given about the extracted features:

\subsection{Sample Entropy (SampEnt)}

Sample entropy is a useful tool for investigating time series signal. It is used for assessing the complexity of a physiological time-series signal such as EMG signal.

SampEnt is the negative natural logarithm of the probability that two sequences similar for $(\mathrm{m})$ points remain similar at the next point, where self matches are not included in 

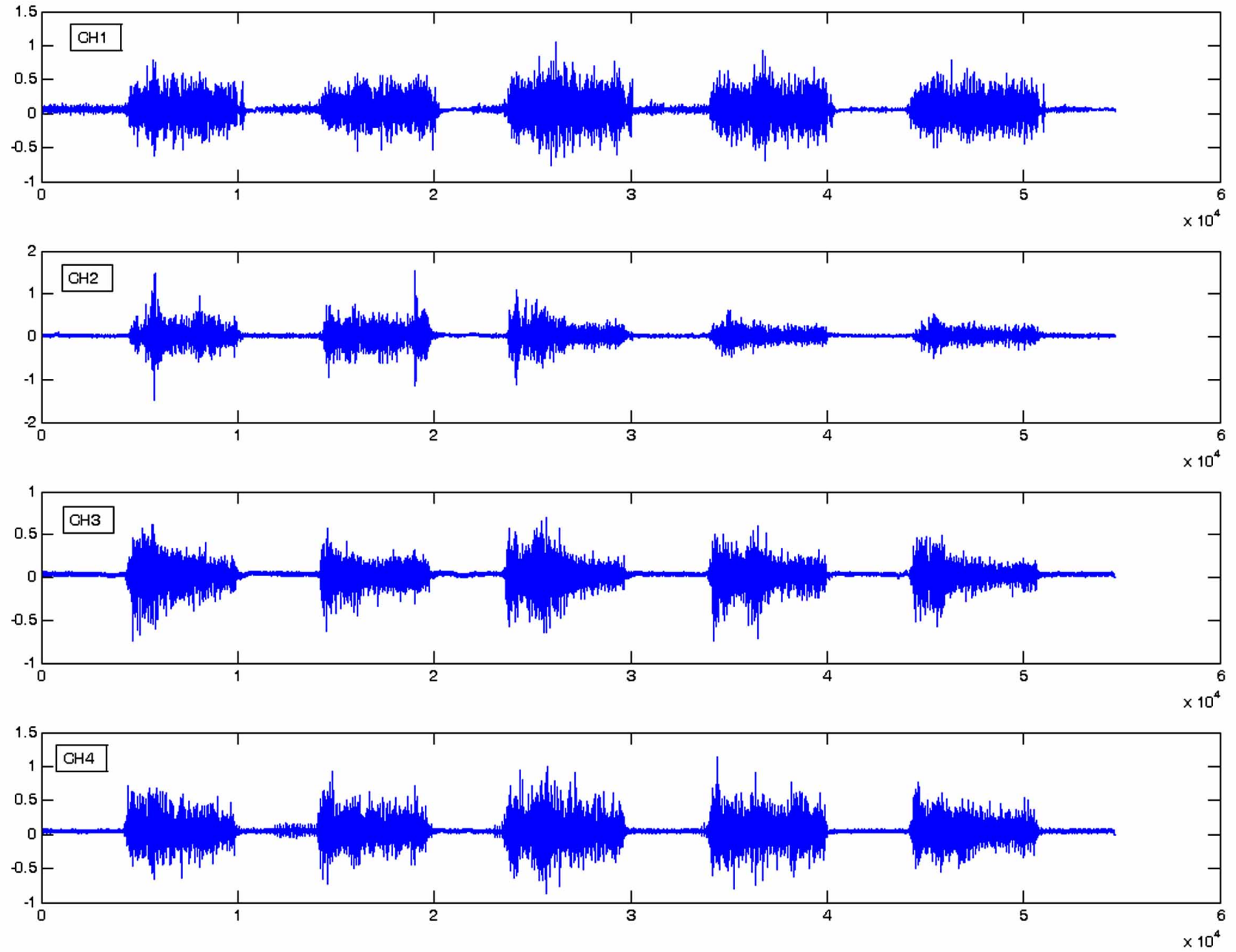

Fig. (3). Four channel sEMG signals were recorded using two EMG data acquisition systems. The subject performs one movement and repeats it five times. The acquired sEMG signal was sampled at $1 \mathrm{KHz}$. The amplitude of the sEMG signal is represented on the $\mathrm{Y}$ axis.

calculating of the probability. SampEnt shows success as a robust feature $[10,11]$. This feature consider as a non linear signal processing method. The SampEnt can be calculated by [11]:

$\operatorname{SampEn}(\mathrm{m}, \mathrm{r}, \mathrm{N})=-\ln \left(\frac{A^{m}(r)}{B^{m}(r)}\right)$

$B$ is the total number of template matches of length $\mathrm{m}$.

$A$ is the total number of forward matches of length $\mathrm{m}+1$.

$A^{m}(r)$ and $B^{m}(r)$ are the probabilities that two series will match for $\mathrm{m}$ and $\mathrm{m}+1$ points.

The SampEnt also can defined as the negative natural logarithm of an estimate of the conditional probability that the patterns of the time series that are similar to each other within a predefined tolerance $(r)$ will remain similar for the next comparison point $[11,12]$.

\subsection{Root Mean Square (RMS)}

It is one of the most popular features used in representation EMG signal. RMS has a robust performance in a noisy environment than other feature [13]. Where $X_{i}$ is the $i$ th sample in segment $i$. $N$ is the number of samples in each segment.

$$
R M S=\sqrt{\frac{1}{N} \sum_{i=1}^{N} x_{i}^{2}}
$$

\subsection{Myopulse Percentage Rate (MYOP)}

The output of this feature is defined as one when the absolute value of the signal is above predefined threshold; otherwise it will be zero [8]. It is defined as:

$$
M Y O P=\sum_{i=1}^{N} f\left(x_{i}\right)
$$


Table 2. Average classification rates using LDA, QDA and K-NN classifiers based on db10 wavelet family at different decomposition levels (DL). The bold numbers represent the highest classification accuracies among the classifiers.

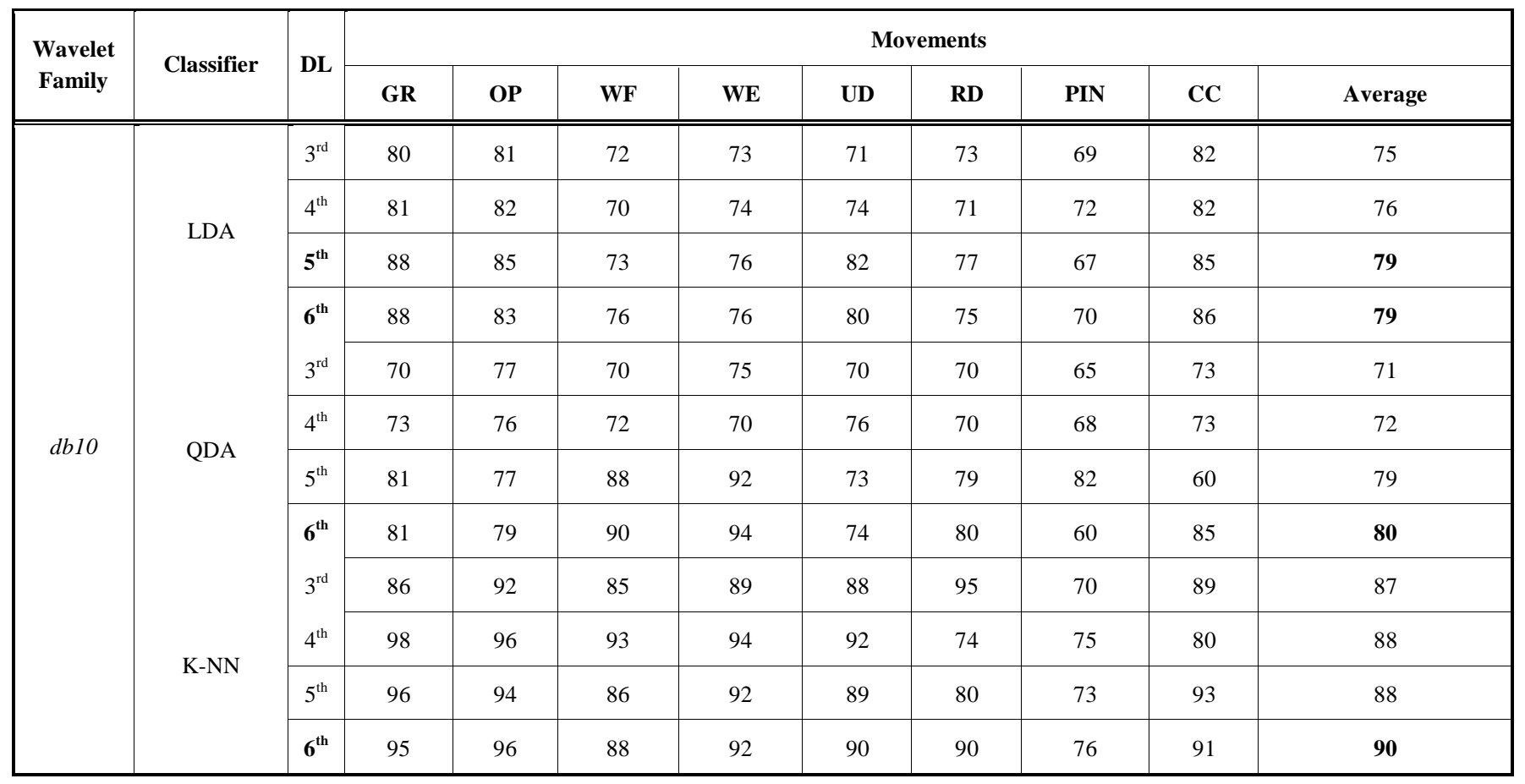

$f(x)=\left\{\begin{array}{l}1 \text { if } x>\text { threshold } \\ 0 \text { if } x<\text { threshold }\end{array}\right.$

\subsection{Difference Absolute Standard Deviation Value (DASDV)}

It is the standard deviation absolute value of the distance between the adjacent samples [14]. Mathematically, it is calculated by:

$D A S D V=\sqrt{\frac{\sum_{i=1}^{N}\left(X_{i+1}-X_{i}\right)^{2}}{N-1}}$

Where $X_{i}$ is the $i$ th sample in segment $i . N$ is the number of samples in each segment.

\subsection{Wilson Amplitude (WAMP)}

This feature is an indicator of firing motor unit action potential (MUAP) that is an indicator of the contraction level. As definition, Wilson amplitude is the number of times that the difference between the signal amplitude among to adjacent segments that exceeds predefined threshold. The benefit of this feature is to reduce the noise effect [8].

$W A M P=\sum_{i=1}^{N} f\left(x_{i}-x_{i+1}\right)$

\subsection{Wavelet Coefficients (WAVELET)}

In last years wavelet analysis becomes a promising tool for many researchers in the field of myoelectric control system and has many applications [15-17]. Wavelet analysis employs a prototype function or a set of basic functions called the mother wavelet. Wavelet transform represents more flexible approach with variable sized window [18]. In this study, Daubechies wavelet family (db10) was employed to extract the wavelet coefficient from the recorded sEMG data.

Choosing the appropriate wavelet family plays a major role in determining the classification rate of a myoelectric control pattern recognition system for a prosthetic hand. However, the ability of DWT to extract features from the signal is dependent on the appropriate choice of the mother wavelet function [19]. In this study, we found that wavelet family db-10 with K-NN classifier gave a high classification rate (preserved most information from EMG signal). The acquired signal is decomposed using Daubechies wavelet family at four different levels: third, fourth, fifth and sixth. Based on the data shown in the Table 2, we also find that increases in the decomposition level of the wavelet family increase the classification rate value.

In total, we analyzed ten subjects, eight classified movements, four channels, and five repetitions of each movement, which results in total of 1600 subsets $(10 \times 8 \times 4 \times 5=1600$ subsets).

The MATLAB (2012a) computational software was used to extract the features from the sEMG signal and for the classification procedure. The data were divided into three sets, $20 \%$ for training, $20 \%$ validation and $60 \%$ for test. For the classification step, three classifiers were implemented: LDA, QDA and k-NN. The coming section provides a brief introduction to the tested classifiers. 
Table 3. Comparison of the LDA, QDA, and K-NN classification algorithms. The bold numbers represent the highest classification accuracies.

\begin{tabular}{|c|c|c|}
\hline Features & Classifier & Classification Rate\% \\
\hline \multirow{3}{*}{ SampEnt } & LDA & 93.23 \\
\hline & QDA & 90.22 \\
\hline & $\mathrm{K}-\mathrm{NN}$ & 91.43 \\
\hline \multirow{3}{*}{$R M S$} & LDA & 92.34 \\
\hline & QDA & 89.44 \\
\hline & $\mathrm{K}-\mathrm{NN}$ & 88.81 \\
\hline \multirow{3}{*}{ MYOP } & LDA & 90.62 \\
\hline & QDA & 88.27 \\
\hline & K-NN & 89.54 \\
\hline \multirow{3}{*}{$D A S D V$} & LDA & 92.22 \\
\hline & QDA & 90.56 \\
\hline & $\mathrm{K}-\mathrm{NN}$ & 90.23 \\
\hline \multirow{3}{*}{$W A M P$} & LDA & 73.44 \\
\hline & QDA & 70.85 \\
\hline & K-NN & 77.44 \\
\hline \multirow{3}{*}{ WAVELET } & LDA & 78.43 \\
\hline & QDA & 80.23 \\
\hline & K-NN & 90.92 \\
\hline \multirow{3}{*}{ SampEnt-RMS } & LDA & 94.82 \\
\hline & QDA & 90.73 \\
\hline & K-NN & 90.15 \\
\hline \multirow{3}{*}{ SampEnt-RMS-MYOP } & LDA & 95.43 \\
\hline & QDA & 92.48 \\
\hline & $\mathrm{K}-\mathrm{NN}$ & 91.63 \\
\hline \multirow{3}{*}{$\begin{array}{c}\text { SampEnt-RMS-MYOP- } \\
D A S D V\end{array}$} & LDA & 98.56 \\
\hline & QDA & 93.42 \\
\hline & $\mathrm{K}-\mathrm{NN}$ & 94.25 \\
\hline
\end{tabular}

\section{CLASSIFICATION ALGORITHMS}

Classifications and groupings of patterns play a key role in solving many problems in a variety of engineering and scientific disciplines such as biology, psychology, medicine, civil engineering, mechanical engineering, data mining, computer vision and artificial intelligence [20-24].

\subsection{Linear Discriminate Analysis (LDA)}

LDA is also recommended as a robust classifier and has been employed in several recent studies $[11,25,26]$. This classifier was chosen because it does not require any parameter adjustment and offers computationally efficient real time operation $[11,27,28]$. The main concept of LDA is to classify the dependence by separating an m-dimensional descriptor space into two areas that are separated by a hyper plane defined by a linear discriminant function. LDA minimizes the distances among the vectors belonging to the same class and maximizes the distances among the class centers [5]. LDA is recommended as the robust classifier and it has been employed in several recent literatures [9]. The classifier was chosen because it does not require any parameter adjustment and computationally efficient real time operation.

\subsection{Quadratic Discriminate Analysis (QDA)}

QDA is also a robust classification technique. It is a nonlinear technique for pattern classification. QDA models the likelihood of each class as a Gaussian distribution, then uses the posterior distributions to estimate the class for a given test point. The Gaussian parameters for each class can be estimated from training points using maximum likelihood (ML) estimation [29]. QDA is mathematically intractable, as the estimates of the covariance matrices for each class become either poorly posed [30]. More information regarding LDA and QDA can be found in the work of Kim and Grouven $[14,31]$.

\subsection{K-Nearest Neighbor (k-NN)}

The k-nearest neighbor $(k-\mathrm{NN})$ classification method is a classical method that is widely used in the field of EMG pattern recognition $[14,32,33]$. The performance of this classifier can be degraded in noisy environments [14]. The mechanism of this classifier is based on three steps: 1- calculate the distances between a query sample and all training samples, 2- choose the K-nearest training samples to the query sample, and 3- assign a class label by applying the majority rule to the $k$ nearest samples [34]. In other words, this classifier predicts the test sample's category according to $\mathrm{k}$ training samples and classifies it to the class with the highest class probability [14].

\section{RESULTS AND DISCUSSION}

The following results were deduced from the data shown in Table 3:

1. SampEnt is a robust feature that gives a high classification rate (higher than $90 \%$ ) for all of the studied classification methods. The highest classification rate of $93.23 \%$ was achieved using the LDA classifier. Other researchers have used this feature to classify different myoelectric patterns and have also reported that it shows robust performance compared with other features $[11,35]$. 


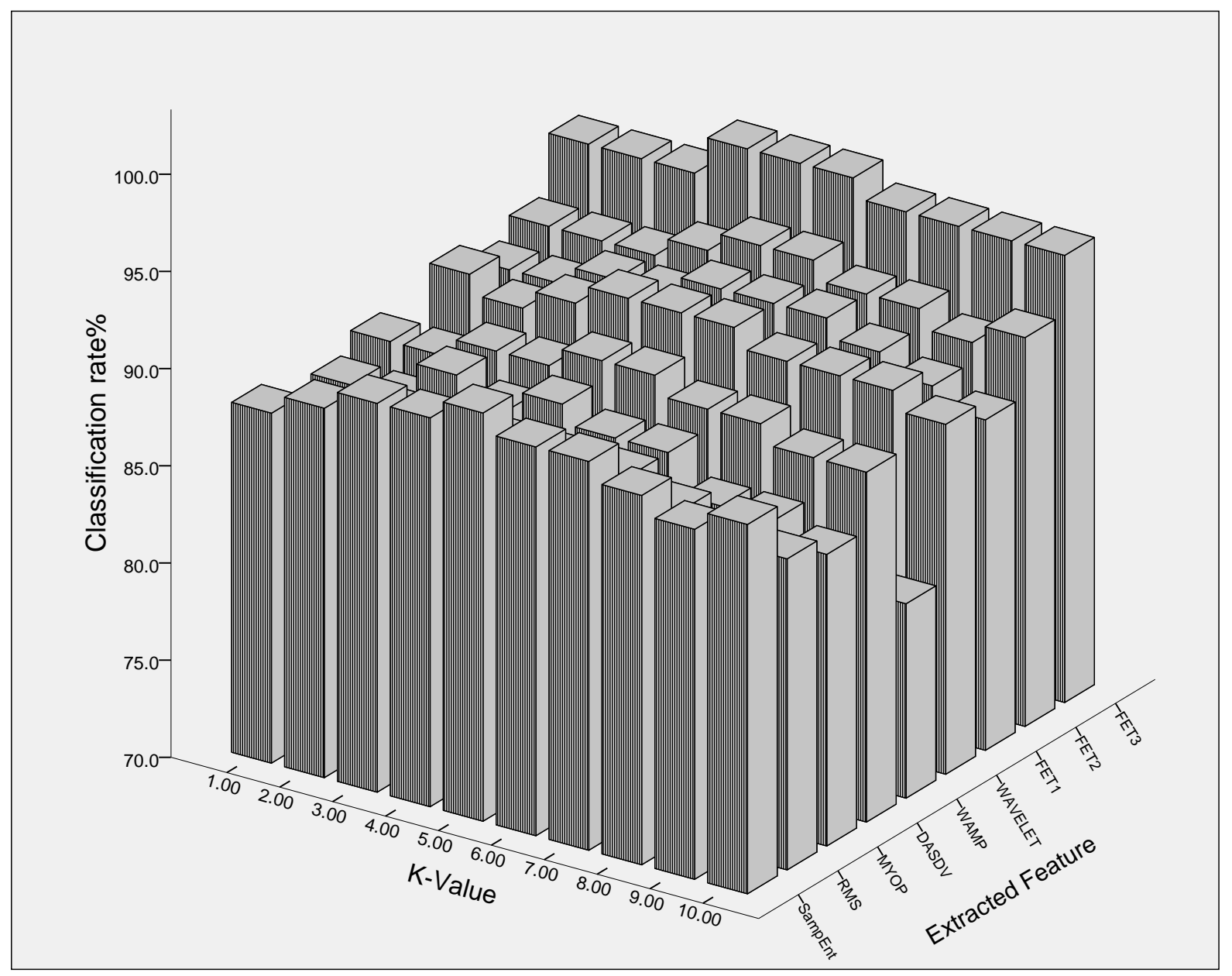

Fig. (4). Classification rates obtained using the K-NN recognition method based on different extracted features. The tested $\mathrm{k}$ values were $\mathrm{k}=1,2 \ldots 10$. The value $\mathrm{k}=5$ was the optimal value for the $\mathrm{k}-\mathrm{NN}$ classifier because it gives highest classification rate.

2. Other interesting result was that the classification accuracy increased with the addition of extracted features. In other words, the performance of a classifier was improved through the implementation of more than one feature.

3. In this paper, we tested three feature combinations: SampEnt-RMS, SampEnt-RMS-MYOP, and SampEnt-RMS-MYOP-DASDV. The highest classification accuracy was achieved using the LDA classifier, and the classification rates obtained using this classifier and the three abovementioned combinations were found to be $94.82 \%, 95.43 \%$, and $98.56 \%$, respectively.

4. For all of the features except the WAVELET, the performance of LDA was better than that of QDA. The WAVELET feature gave accuracies of $78.43 \%$ and $80.23 \%$ for LDA and QDA, respectively.

5. For the K-NN classifier, the best average classification accuracy was $94.25 \%$, and this rate was obtained using the SampEnt-RMS-MYOP-DASDV set. The next higher accuracies were obtained using SampEntRMS-MYOP and SampEnt, which gave a classification rate of $91 \%$.

The choice of the optimal k-value for the k-NN classifier plays a role in the determination of the performance of the $\mathrm{K}-\mathrm{NN}$ recognition algorithm. The result of this investigation, which is clarified in Fig. (4), demonstrates that $\mathrm{k}=5$ gave the best performance of K-NN in most cases.

\section{CONCLUSION}

In this study, we succeeded to achieve $98.56 \%$ classification rate by using the LDA classifier based on SampEntRMS-MYOP-DASDV feature set. This result is considered to be a high classification rate in case eight hand motions are recognized based on four sEMG signals.

More classification methods should be implemented to classify more different patterns of hand motions and to 
achieve higher classification rates. General regression neural network (GRNN), support vector machine (SVM) classifiers are recommended for future work. One more important factor should be considered when designing a myoelectric control system: computational time. Increasing the number of sEMG channels will improve the accuracy rate but will increase the calculation time of feature extraction and, of course, the classification time. A feature combination with a long computation time should be avoided when selecting the feature combination. Other time domain and frequency domain features should be extracted from the recorded EMG.

\section{CONFLICT OF INTEREST}

The authors confirm that this article content has no conflict of interest.

\section{ACKNOWLEDGEMENTS}

Declared none.

\section{REFERENCES}

[1] R. N. Khushaba, S. Kodagoda, D. Liu, and G. Dissanayake, "Muscle computer interfaces for driver distraction reduction", Computer Methods and Programs in Biomedicine, vol. 110, no. 2, pp. 137-149, 2012.

[2] D. K. Kumar, N. D. Pah, and A. Bradley, "Wavelet analysis of surface electromyography to determine muscle fatigue," IEEE Transactions on Neural Systems and Rehabilitation Engineering, vol. 11, no. 4, pp. 400-406, 2003.

[3] H. Huang, J. Y. Guo, and H. J. Chen,"Ant colony optimizationbased feature selection method for surface electromyography signals classification", Computers in Biology and Medicine, vol. 42, no. 1, pp. 30-38, 2012.

[4] J. Kilby, H. Gholam, "Wavelet analysis of surface electromyography signals," In: Annual International Conference of the IEEE Engineering in Medicne and Biology Society, San Francisco-USA, 2004.

[5] M. Hariharan, C.Y. Fook, R. Sindhu, B. Ilias, and S. Yacoob, "A comparative study of wavelet families for classification of wrist motions," Computers \& Electrical Engineering, vol. 38, no. 6, pp. 1798-1807, 2012

[6] C. Eugenio, N. M .L. Orosco, and F. Sciascio, "Bispectrum-based features classification for myoelectric control," Biomedical Signal Processing and Control, vol. 2, no. 8, pp. 153-168, 2013.

[7] S. Leif, and L. Pablo, "Bioelectrical signal processing in cardiac and neurological applications," Elsevier Academic Press, 2005.

[8] A. Phinyomark, P. Phukpattaranont, and C. Limsakul, "Feature reduction and selection for EMG signal classification," Expert Systems with Applications, vol. 39, no. 8, pp. 7420-7431, 2012.

[9] M. A. Oskoei, and H. Hu. "A survey-Myoelectric control systems", Biomedical Signal Processing and Control, vol. 2, pp. 275-294, 2012.

[10] Z Z . Xu, and Z. Ping," Sample entropy analysis of surface EMG for improved muscle activity onset detection against spurious background spikes", Journal of Electromyography and Kinesiology, vol. 22, no. 6, pp. 901-907, 2012.

[11] Phinyomark A, F. Quaine, S. Charbonnier, C. Servier, F. Trapin Bernard and Y. Lauilluh, "EMG feature evaluation for improving myoelectric pattern recognition robustness," Expert Systems with Applications, vol. 40, no. 12, pp. 4832-4840, 2013.

[12] J. Richman, and J. Moorman," Physiological time-series analysis using approximate entropy and sample entropy," American Journal of Physiology: Heart and Circulatory Physiology, vol. 6, pp. 278, 2000 .
[13] A. Phinyomark, and P. Phukpattaranont,"A novel feature extraction for robust EMG pattern recognition," Journal of Computing, vol. 1, no. 1, pp. 71-80, 2009.

[14] K. S. Kim, H. H. Moon, and C. S. Mun, "Comparison of k-nearest neighbor, quadratic discriminant and linear discriminant analysis in classification of electromyogram signals based on the wrist-motion directions", Current Applied Physics, vol. 11, no. 3, pp. 740-745, 2011.

[15] P. Flandrin, "Wavelet analysis and its applications," Academic Press, San Diego, 1999.

[16] S . Mallat, "A wavelet tour of signal processing," Academic Press, Stanford, 2008.

[17] G. Amara, "An introduction to wavelet," IEEE Computer Sciences and Engineering, vol. 2, no.2, pp. 17, 1995.

[18] M. Misiti ,"Wavelet toolboxtor use with MATLAB- user's guide," IEEE Computational Science and Engineering, vol. 2, no. 2, 1995.

[19] D. K. Kumar, N. D. Pah, and A. Bradley, "Wavelet analysis of surface electromyography to determine muscle fatigue", IEEE Transactions on Neural Systems and Rehabilitation Engineering, vol. 11, no. 4, pp. 400-406, 2003.

[20] P. Brezillon, K. W. Chau, and L. Utkin," Multilayer ensemble pruning via novel multi-sub-swarm particle swarm optimization", Journal of Universal Computer Science, vol. 15, no. 4, pp. 840$858,2009$.

[21] Z. K. Huang, and K. W. Chau, "A new image thresholding method based on gaussian mixture model", Applied Mathematics and Computation, vol. 205, no. 2, pp. 899-907, 2008

[22] R. Taorminaa, "Artificial Neural Network simulation of hourly groundwater levels in a coastal aquifer system of the Venice lagoon," Engineering Applications of Artificial Intelligence, vol. 25, no. 8, pp. 1670-1676, 2012.

[23] K. W. Chau, "Application of a PSO-based neural network in analysis of outcomes of construction claims", Automation in Construction, vol. 16, pp. 643-646, 2012.

[24] C. L. Wu, K. W. Chau, and Y. S. Li ,"Predicting monthly streamflow using data-driven models coupled with datapreprocessing techniques", Water Resources Research, vol. 45, no. 8, 2009.

[25] A. J .Young, L .J. Hargrove, and T. A. Kuiken, "Improving myoelectric pattern recognition robustness to electrode shift by changing interelectrode distance and electrode configuration", IEEE Transactions on Biomedical Engineering, vol. 59, no. 3, pp. 645-652, 2012.

[26] D. Tkach, H. Huang and T. A. Kuiken, "Study of stability of domain time features for electromyographic pattern recognition", Journal of Neuroengineering and Rehabilitation, vol. 7, no. 21, 2010 .

[27] K. Paul, E. Kevi, and P. M. Book," Fluctuating emg signals: investigating long-term effects of pattern matching algorithms," Proceedings of $32^{\text {nd }}$ annual international conference of the IEEE engineering in medicine and biology society, pp. 6357-6360, 2010.

[28] K. Englehart, B. Hudgins, P. A. Parker, and M. Stevenson, "A wavelet-based continuous classification scheme for multifunction myoelectric control," IEEE Transaction on Biomedical Engineering, vol. 48, no. 3, pp. 302-311, 2001.

[29] S. Srivastava , Bayesian Quadratic Discriminant Analysis. Journal of Machine Learning Research 2007 August 2013]; 1277-1305]. Available from: http://people.ee.duke.edu/ lcarin/SrivastavaGuptaFrigyikBDA.pdf

[30] Wang J, K. N. Plataniotis, J. Lu and A. N. Venetsanopoulos, "Kernel quadratic discriminant analysis for small sample size problem", Journal of Pattern Recognition, vol. 41, no. 5, pp. 1528$1538,2008$.

[31] U. Grouven, F. Bergel, and A. Schultz, "Implementation of linear and quadratic discriminant analysis incorporating costs of misclassification", Computer Methods and Programs in Biomedicine, vol. 49, no. 1, pp.5 5-60, 1996.

[32] X. Chen, and Z. J .Wang," Pattern recognition of number gestures based on a wireless surface EMG system", Biomedical Signal Processing and Control, vol. 8, no. 2, pp. 184-192, 2013. 
[33] A . Subasi, "Classification of EMG signals using PSO optimized SVM for diagnosis of neuromuscular disorders", Computers in Biology and Medicine, vol. 43, no. 5, pp. 576-586, 2013.

[34] J. Toyama, M. kudo, and H. Imai, "Probably correct k-nearest neighbor search in high dimensions", Journal of Pattern Recognition, vol. 43, no. 4, pp. 1361-1372, 2010.
[35] X. Zhang, "Sample entropy analysis of surface EMG for improved muscle activity onset detection against spurious background spikes", Journal of Electromyography and Kinesiology, vol. 22, no. 6, pp. 901-907, 2012.

Received: January 16, 2014

(C) AlOmari and Liu; Licensee Bentham Open.

This is an open access article licensed under the terms of the Creative Commons Attribution Non-Commercial License (http://creativecommons.org/licenses/by-nc/3.0/) which permits unrestricted, non-commercial use, distribution and reproduction in any medium, provided the work is properly cited. 